\title{
Methods for generation of monoclonal antibodies to the very small drug hapten, 5-benzimidazolecarboxylic acid
}

\author{
E. Moran ${ }^{\mathrm{a}, *}$, M. O’Keeffe ${ }^{\mathrm{b}}$, R. O’Connor ${ }^{\mathrm{a}}$, A.M. Larkin ${ }^{\mathrm{a}}$, P. Murphy ${ }^{\mathrm{a}}$, M. Clynes ${ }^{\mathrm{a}}$ \\ ${ }^{\mathrm{a}}$ National Cell and Tissue Culture Centre, Dublin City University, Glasnevin, Dublin 9, Ireland \\ ${ }^{\mathrm{b}}$ Food Safety Department, The National Food Centre, Teagasc, Dunsinea, Castleknock, Dublin 15, Ireland
}

Received 25 February 2002; received in revised form 7 June 2002; accepted 14 August 2002

\begin{abstract}
Drug-specific monoclonal antibodies (MAbs) were produced against the very small drug hapten (162.15 Da), 5benzimidazolecarboxylic acid, an analogue of 2-(4-Thiazolyl)benzimidazole (TBZ) but lacking the thiol group. TBZ is widely used as a broad-spectrum anthelmintic in various animal species and humans and also as a food preservative and agricultural fungicide. The anti-5-benzimidazolecarboxylic acid antibodies produced have potential use for extraction and/or detection of protein-bound residue forms of TBZ.

Three in vivo immunisation regimes (with combinations of two related small drug haptens and two different adjuvants/ carrier molecules) and an in vitro immunisation procedure using a combination of three related unconjugated small drug haptens were investigated. Specificity for the hapten immunogen/s was initially determined using two different ELISA procedures. BIACORE analysis, in conjunction with drug binding inhibition studies, was used to confirm the specificity of a small number of selected clones.

In vivo immunisation with a drug molecule conjugated to a lipopeptide/T-cell epitope, which acts both as a carrier molecule and an adjuvant was the most useful of the methods tested for the production of specific MAbs to a typically very small hapten with low immunogenic properties.
\end{abstract}

(C) 2002 Elsevier Science B.V. All rights reserved.

Keywords: 2-(4-Thiazolyl)benzimidazole; 5-Benzimidazolecarboxylic acid; Small haptens; Monoclonal antibodies; Lipopeptide/T-cell epitope

\section{Introduction}

2-(4-Thiazolyl)benzimidazole (TBZ) is widely used as a broad-spectrum anthelmintic in various animal species and humans and also as a food

\footnotetext{
* Corresponding author. BioResearch Ireland, Enterprise Ireland, Glasnevin, Dublin 9, Ireland. Tel.: +353-1-808-2696.

E-mail address: moranl@biores-irl.ie (E. Moran).
}

preservative and agricultural fungicide (Coulet et al., 1998a). Following a study of the relevant literature which indicated that protein-bound tissue residues of TBZ possess an intact benzimidazole ring structure (Coulet et al., 1998a,b), the purpose of this work was to choose a drug (hapten) that could be bound to a suitable carrier protein (to increase its immunogenicity) and allow the ring structure of benzimidazole to be exposed as a major antigenic site. Previously, Brandon et al. (1992) reported the production of 
monoclonal antibodies (MAbs) cross-reacting with TBZ and its major metabolite 5-hydroxythiabendazole using the immunogens 2- and 5-Succinamidothiabendazole. During this study, the TBZ parent compound plus its four analogues were used as immunogens, i.e. 5-hydroxybenzimidazole carbamate, 5-hydroxythiabendazole, 5-benzimidazolecarboxylic acid and benzimidazole.

The possibility of raising MAbs with a high affinity for the target antigen is greatly reduced for haptens with a molecular weight of $<300 \mathrm{Da}$ and MAbs to such small-sized haptens can only be generated using the hapten conjugated to a carrier molecule (Chappey et al., 1994). The selection of immunogens and thereafter the selection of a carrier molecule and method/s of conjugation of the immunogens is critical for the successful production of specific MAbs. The coupling site of the carrier molecule in addition to the length of the linker group have been shown to be important considerations which need to be taken into account in terms of the region of the molecule recognised and hapten specificity of resultant MAbs (Chappey et al., 1992; Tuomola et al., 2000). Hence, the importance of the development and optimisation of conjugation methods to carrier proteins and investigation of adjuvant systems designed specifically to aid induction of high levels of specific antibodies to the hapten (Hoffmann et al., 1997).

During this study, three in vivo immunisation regimes and one in vitro procedure were investigated. The in vitro immunisation procedure has the combined advantages of not necessarily requiring a carrier protein attached to a small hapten, use of very small quantities of immunogen (Holley, 1998) and nonclearance of the immunogen via the renal system, as seen with in vivo procedures.

Reports of increased antigen uptake by antigenpresenting cells when haptens are conjugated to a modified form of a carrier protein, i.e. cationised BSA, prompted its adoption for conjugation to one of the haptens 5-hydroxybenzimidazole carbamate for use in one of the in vivo immunisation regimes (Apple et al., 1988). More recent reports by Hoffmann et al. (1997) and Baier et al. (2000) on the use of a lipopeptide $\left(\mathrm{Pam}_{3} \mathrm{Cys}\right)$ coupled to a $\mathrm{T}_{\mathrm{h}}$-cell epitope (SFISEAIIHVLHSRHPG), both as a carrier molecule and as an adjuvant for the production of
MAbs to low-molecular weight toxins, also led to the use of both admixtures of hapten and lipopeptide $/ \mathrm{T}_{\mathrm{h}^{-}}$ cell epitope and hapten conjugated to lipopeptide/ $/ \mathrm{T}^{-}$ cell epitope for two further in vivo immunisation regimes.

One particularly successful method was identified (i.e., the conjugation of the hapten to the lipopeptide/ $\mathrm{T}_{\mathrm{h}}$-cell epitope) for the production of hapten-specific MAbs that can now be further optimised and applied to the production of specific MAbs to a wide range of very small haptens which may have applications in studies related to food and health safety.

\section{Materials and methods}

2-(4-Thiazolyl)benzimidazole (TBZ), 5-benzimidazolecarboxylic acid, benzimidazole, 10-decanoic acid, 2-benzimidazolepropionic acid, 1-Ethyl-3-(3dimethylaminopropyl)carbodiimide (EDC) hydrochloride (E-7750), DMSO (D-5879) and Freund's complete and incomplete adjuvants were all purchased from Sigma-Aldrich (Ireland). 5-Hydroxybenzimidazole carbamate was a gift from Dr. Pierre Galtier of INRA, Toulouse, France. Merck Sharp and Dohme (Rahway, NJ, USA) provided 5-hydroxythiabendazole. Imject Supercarrier Immune Modulator, BSA carrier protein (cBSA) (Cat. No. 77165), sulpho-NHS (Cat No. 24510) and the BCA protein assay kit (Cat. 23225) were purchased from Pierce Chemical (Rockford, IL, USA). Lipopeptide/ $\mathrm{T}_{\mathrm{h}}$-cell epitope carrier protein/adjuvant $\left(\mathrm{Pam}_{3} \mathrm{Cys}-\mathrm{T}_{\mathrm{H}}\right)$ and the custom-produced 5-benzimidazolecarboxylic acid/Pam ${ }_{3} \mathrm{Cys}_{\mathrm{H}} \mathrm{T}_{\mathrm{H}}$ conjugate were both purchased from EMC microcollections ${ }^{\circledR}$, Sindelfinger Str. 3, 72070 Tubingen, Germany. MaxiSorp ELISA plates and CovaLink NH ELISA plates were obtained from Nunc (DK-4000 Roskilde, Denmark).

\subsection{Preparation of immunogens}

\subsubsection{Immunogen 1: 5-hydroxybenzimidazole carbamate/cBSA}

Conjugation of 5-hydroxybenzimidazole carbamate to the carrier protein cBSA was carried out by EDC chemistry which reacted with the available carboxyl group on 5-hydroxybenzimidazole carbamate to form an amine-reactive site for conjugation 
to the carrier protein. The conjugate was purified by dialysis followed by protein analysis. Briefly, a vial of Supercarrier cBSA protein was reconstituted with 200 $\mu \mathrm{l}$ of dist $\mathrm{H}_{2} \mathrm{O}$ (2 $\mathrm{mg}$ Supercarrier cBSA/vial). $2 \mathrm{mg}$ of hapten (5-OH-benzimidazole carbamate) was dissolved in $100 \mu \mathrm{l}$ of DMSO and $400 \mu \mathrm{l}$ of conjugation buffer consisting of $0.1 \mathrm{M} 2$-( $N$-Morpholino)-ethanesulfonic acid (MES), $0.9 \mathrm{M} \mathrm{NaCl}, 0.02 \% \mathrm{NaN}_{3}, \mathrm{pH}$ 4.7 was slowly added. It was recommended (by the suppliers of the Supercarrier) not to use a concentration greater than $30 \%$ DMSO in the final conjugation solution since the carrier protein may be irreversibly denatured. A hapten solution $(2 \mathrm{mg})$ of $500 \mu \mathrm{l}$ was added to $200 \mu \mathrm{l}$ of carrier protein solution (2 mg).

EDC $(10 \mathrm{mg})$ was dissolved in $300 \mu \mathrm{l}$ of $\mathrm{H}_{2} \mathrm{O}$ and $100 \mu \mathrm{l}$ was added to the carrier/hapten solution. The reaction was allowed to continue for $2 \mathrm{~h}$ at RT. This solution was then dialysed overnight against a purification buffer $(0.083 \mathrm{M}$ sodium phosphate, $0.9 \mathrm{M}$ $\mathrm{NaCl}, \mathrm{pH}$ 7.2). A protein assay using the Pierce BCA kit was used to determine protein concentration and aliquots $(100 \mu \mathrm{l})$ were stored at $-20{ }^{\circ} \mathrm{C}$ prior to immunisation of $\mathrm{BALB} / \mathrm{c}$ mice. A spectrum of the cBSA alone conjugated to EDC (no hapten) was compared to a spectrum of 5-OH-benzimidazole carbamate/cBSA conjugated with EDC between the wavelengths of 200-400 $\mathrm{nm}$. Likewise, the spectrum of 5-OH-benzimidazole carbamate in PBS/DMSO was compared to PBS/DMSO alone over the same wavelength range.

\subsubsection{Immunogen 2: admixture of drugs $/ \mathrm{Pam}_{3} \mathrm{Cys}-\mathrm{T}_{H}$}

An admixture of 5-hydroxybenzimidazole carbamate conjugated to cBSA plus free unconjugated 5benzimidazolecarboxylic acid and the adjuvant/carrier $\mathrm{Pam}_{3} \mathrm{Cys}_{\mathrm{H}} \mathrm{T}_{\mathrm{H}}$ was prepared by sonication in $0.9 \%$ $\mathrm{NaCl}$ prior to use.

\subsubsection{Immunogen 3: 5-benzimidazolecarboxylic acid/ $\mathrm{Pam}_{3} \mathrm{Cys}-\mathrm{T}_{H}$ conjugate}

The conjugate of $\mathrm{Pam}_{3} \mathrm{Cys}-\mathrm{T}_{\mathrm{H}}$ and 5-benzimidazolecarboxylic acid (custom prepared by EMC microcollections ${ }^{\circledR}$ ) was produced by conjugation of the carboxylic group of 5-benzimidazolecarboxylic acid to the $\varepsilon$-amino group of an additional lysine at the $\mathrm{N}$-terminus of the $\mathrm{T}_{\mathrm{H}}$ sequence (SFISEAIIHVLHSRHPG).
Immunogens 1, 2 and 3 were used for the in vivo immunisation regimes.

2.1.4. Immunogen 4: combination of free drugs, i.e. TBZ, 5-hydroxythiabendazole and benzimidazole

A combination of the parent drug (TBZ) and its main metabolite 5-hydroxythiabendazole and benzimidazole was used as immunogens for the in vitro immunisation procedure. No adjuvant or carrier protein was used for this immunisation protocol which involved the use of a specialised in vitro immunisation kit obtained from Immune Systems (Paignton, UK). TBZ and 5-hydroxythiabendazole were dissolved in DMSO/ethanol (1:3) and benzimidazole was dissolved in $\mathrm{DMSO} / \mathrm{H}_{2} \mathrm{O}$ (1:5); all drug preparations being filter sterilised prior to use.

\subsection{Immunisation regimes}

\subsubsection{In vivo immunisation protocol with immunogen 1}

Using four BALB/c mice per immunogen, three separate in vivo immunisation procedures were performed. A primary immunisation with 5-hydroxybenzimidazole carbamate conjugated to cBSA (60$100 \mu \mathrm{g}$ protein $/ 500 \mu \mathrm{l}$ injection) was administered intraperitoneally (i.p.) in Freund's complete adjuvant and subsequent booster immunisations (four) in Freund's incomplete adjuvant were similarly administered at 4- to 6-week intervals prior to the fusion procedure.

\subsubsection{In vivo immunisation protocol with immunogen 2 \\ Using the admixture of 5-hydroxybenzimidazole} carbamate/cBSA conjugate plus free unconjugated 5benzimidazolecarboxylic acid and $\mathrm{Pam}_{3} \mathrm{Cys}_{\mathrm{H}} \mathrm{T}_{\mathrm{H}}$ (as an adjuvant), one primary and three booster immunisations were given as above. $25-23 \mu \mathrm{g}$ of 5-Hydroxybenzimidazole carbamate/cBSA ( $50 \mu 1$ volume) and approximately $50 \mu \mathrm{g}$ of 5-benzimidazolecarboxylic acid (50 $\mu \mathrm{l}$ volume) were combined by sonication with $100 \mu \mathrm{l}$ of $\mathrm{Pam}_{3} \mathrm{Cys}_{\mathrm{H}} \mathrm{T}_{\mathrm{H}}$ /injection.

\subsubsection{In vivo immunisation protocol with immunogen 3}

A similar immunisation procedure (i.e., one primary and three booster immunisations) was undertaken using the custom-prepared conjugate of 
$\mathrm{Pam}_{3} \mathrm{Cys}_{\mathrm{H}} \mathrm{T}_{\mathrm{H}}$ and 5-benzimidazolecarboxylic acid ( $\sim 50-\mu$ g conjugate/50- $\mu$ l injection).

During the immunisation schedule and prior to the fusion procedure, polyclonal serum obtained from the tail-bleeds of animals (taken following the third booster injections) was used to develop the most suitable ELISA screening method to monitor reactivity with the drug used as the immunogen and also crossreactivity with other related drugs. Serum from the tail-bleed of a non-immunised mouse was used as a negative control. All animal procedures were performed under license, in compliance with the Cruelty To Animal Act 1876 (European Community Directive, 86/609/EC).

\subsubsection{In vitro immunisation protocol with immunogen 4}

There were three separate stages in the in vitro immunisation process. Firstly, a monolayer of proprietary support cells (supplied with the in vitro immunisation kit) was established and primed with filter-sterilised antigen/s. Fifteen micrograms of the immunogens TBZ, 5-hydroxythiabendazole and benzimidazole were added in $10 \mu \mathrm{l}$ each of the solvents described above (i.e., overall drug concentration of $1.5 \mu \mathrm{g} / \mathrm{ml}$ of culture medium) to the support cells. The flask was left undisturbed for $48 \mathrm{~h}$ at $37{ }^{\circ} \mathrm{C}, 5 \%$ $\mathrm{CO}_{2}$. At the end of this time, the support cells were washed with a support medium supplied with the kit and splenocytes from a 7-week-old BALB/c mouse were added. Sterile antigen was added as above and following the addition of proprietary immunisation medium, the flask was incubated undisturbed for 3 days at $37{ }^{\circ} \mathrm{C}, 5 \% \mathrm{CO}_{2}$. After this time, the primed splenocyte culture was ready for fusion with myeloma cells.

\subsection{Fusion procedure}

Fusion of murine spleen cells and SP2 mouse myeloma cells was performed in all cases according to a modification of the method of Kohler and Milstein (1975). Fused cells were plated in $8 \times 48$ well plates in a post-plating medium as previously described (Moran et al., 1998). Fusion efficiency was defined as the percentage of total wells (i.e., 384 wells) containing one or more hybridoma clones. ELISA, as outlined below, assessed supernatants from the resultant hybridomas for specific $\mathrm{MAb}$ production. Hybridomas chosen for further studies were cloned twice by limiting dilution as previously described (Moran et al., 1998) to ensure monoclonality.

\subsection{Hybridoma screening methods}

Two different types of ELISA procedures were assessed (initially using polyclonal serum from tail bleeds of immunised animals) for preliminary largescale screening/identification of drug-specific hybridoma supernatants.

(a) MaxiSorp ELISA plates (Nunc) were used for screening the supernatants from hybridomas raised to the drug/carrier protein conjugate used for immunogen 1 (i.e., 5-hydroxybenzimidazole carbamate conjugated to cBSA). The polystyrene surface of these plates (with high affinity for polar groups) is excellent for noncovalently binding proteins and the drug is bound to the plate surface via the carrier protein, cBSA.

MaxiSorp plates were also used for the screening of hybridomas raised to the custom-prepared conjugate of 5-benzimidazolecarboxylic acid/ $\mathrm{Pam}_{3} \mathrm{Cys}_{\mathrm{H}} \mathrm{T}_{\mathrm{H}}$ (immunogen 3). Briefly, a modification of the method of Hoffman et al. (1990) was used to coat the ELISA plates. The plates were coated with 5-benzimidazolecarboxylic acid/ $\mathrm{Pam}_{3} \mathrm{Cys}_{\mathrm{H}} \mathrm{T}_{\mathrm{H}}$ or with $\mathrm{Pam}_{3} \mathrm{Cys}_{\mathrm{H}}$ alone in a carbonate buffer of $\mathrm{pH} 9.3$ at a concentration of $5 \mu \mathrm{g} / 100 \mu \mathrm{l} /$ well and were incubated at 37 ${ }^{\circ} \mathrm{C}$ for $2 \mathrm{~h}$ and at $4{ }^{\circ} \mathrm{C}$ overnight. Following the removal of excess immunogen, the plates were washed once with PBS (pH 7.5) and $100 \mu 1$ of blocking buffer $(1 \% \mathrm{BSA} w / \mathrm{v}$ in PBS) was added for $2 \mathrm{~h}$ at RT. Upon removal of the blocking buffer, the plates were washed once with PBS and $50 \mu \mathrm{l}$ of samples (neat hybridoma supernatants) was added and incubated at $4{ }^{\circ} \mathrm{C}$ overnight. The following day, the plates were washed three times with washing buffer (PBS/0.2\% Tween 20) and $100 \mu 1 /$ well of secondary antibody consisting of alkaline phosphatase-conjugated rabbit anti-mouse IgG (Dako, Denmark, Cat. No. D0314) diluted $1 / 1000$ in $1 \%$ BSA w/v in PBS was added for $1.5 \mathrm{~h}$ at $37{ }^{\circ} \mathrm{C}$. The plates were then washed three times as before and $100 \mu \mathrm{l} /$ well of substrate consisting of $1 \mathrm{mg} / \mathrm{ml} p$-nitrophenyl phosphate (PNPP, Sigma, 104-0) in $0.1 \mathrm{M}$ glycine, $1 \mathrm{mM} \mathrm{MgCl} 2,1 \mathrm{mM} \mathrm{ZnCl}$, $\mathrm{pH} 10.4$ was added for $30 \mathrm{~min}$ at $37^{\circ} \mathrm{C}$. The reaction 
was stopped with $2 \mathrm{~N} \mathrm{NaOH}(25 \mu \mathrm{l} /$ well $)$ before reading absorbance values on a Titerex ELISA plate reader at $405 \mathrm{~nm}$.

(b) CovaLink NH ELISA plates (Nunc) have a modified polystyrene surface grafted with $\mathrm{NH}$ functional groups available for the covalent binding of nonconjugated drugs having free carboxylic groups. Formation of amide bonds between carboxylic acids and amines is generated by carbodiimide (EDC) which activates the carboxylate by forming $O$-acylurea. It has been demonstrated that a large hydrolysisresistant active ester can be made by the addition of $N$ hydroxysuccinimide (NHS). The $O$-acylurea-activated ester reacts with the NHS, forming a more stable succinimidyl-activated ester compound (Staros et al., 1986). Covalent binding, unlike simple physical binding, may orientate the immobilised molecules in a defined way on the solid phase. Also, covalent binding can immobilise small molecules that only bind weakly or not at all by physical adsorption, e.g. small hapten molecules such as peptides and the drugs used as immunogens for this work (Nunc Technical Literature).

The free drugs which were conjugated to these plates for the screening of hybridoma supernatants were the TBZ analogues, 5-benzimidazolecarboxylic acid and 2-benzimidazolepropionic acid. Instead of using CovaLink wells with no drug present as negative control wells, it was considered technically more appropriate that wells should be "capped" with a drug completely unrelated to the test drug, i.e. 10-hydroxydecanoic acid. Both the test drug and the "capping" drug were applied to the wells in two unrelated and separate successive operations in order to effectively block all the $\mathrm{NH}$ groups on the CovaLink plates. These steps acted to overcome background readings that would be obtained on wells without "capping" and also false positives that might be obtained on wells with test drug if all $\mathrm{NH}$ groups in these wells were not effectively blocked.

This method of screening was used for assessing supernatants from hybridomas resulting from immunisation regimes with immunogens $1,2,3$ and 4 . Briefly, 5-benzimidazolecarboxylic acid (in $\mathrm{H}_{2} \mathrm{O}$ / DMSO, 1:1.5) and 10-hydroxydecanoic acid (in $\mathrm{H}_{2} \mathrm{O} /$ DMSO, 1:1.5) were made up to appropriate final concentrations in $\mathrm{H}_{2} \mathrm{O}$ containing $1.5 \mathrm{mM}$ sulpho- $N$ hydroxysuccinimide (sulpho-NHS) to give a final concentration of $25 \mu \mathrm{g} / 100 \mu \mathrm{l} / \mathrm{well}$ of each drug. 50 $\mu \mathrm{l}$ of freshly prepared EDC $(3.0 \mathrm{mM})$ was added to each well to start the binding reaction and the plates were incubated for $90 \mathrm{~min}$ at RT and then washed three times with CovaBuffer $(2 \mathrm{M} \mathrm{NaCl}, 40 \mathrm{mM}$ $\mathrm{MgSO}_{4.7} \mathrm{H}_{2} \mathrm{O}, 0.05 \%$ Tween 20/PBS, pH 7.4). This procedure was repeated and the plates were incubated for $60 \mathrm{~min}$ at RT to facilitate "capping" of $\mathrm{NH}$ groups. Test supernatants were added to the plates for 1.5-2.0 $\mathrm{h}$ at RT. Plates were again washed three times with CovaBuffer as before and $100 \mu$ of secondary antibody consisting of HRP-conjugated goat anti-mouse IgG (Dako, Cat No. P0447 diluted $1 / 2000$ in CovaBuffer) was added for $1.5 \mathrm{~h}$ at RT. The plates were then washed three times as before and 200 $\mu \mathrm{l} /$ well of substrate $o$-phenylenediamine dihydrochloride (OPD, Sigma, P-9187) was added and incubated in the dark for $30 \mathrm{~min}$ at RT. The reaction was stopped with $3 \mathrm{M} \mathrm{HCl}(50 \mu \mathrm{l} /$ well $)$ before reading absorbance values on a Titerex ELISA plate reader at $492 \mathrm{~nm}$.

Analysis of a small number of supernatants (selected on the basis of the CovaLink NH ELISA results for hybridoma supernatants reacting with immunogen 2 and the MaxiSorp ELISA results for hybridoma supernatants reacting with immunogen 3) was carried out using a BIACORE $3000^{\mathrm{TM}}$ biosensor (Biacore, Stevenage, Herts. UK) according to a modification of the method of Quinn et al. (1997). Drug inhibition studies with free 5-benzimidazolecarboxylic acid were also carried out in conjunction with the BIACORE analysis for assessment of drug specificity of supernatants from hybridomas raised to immunogen 3 .

\section{Results}

\subsection{Structures of immunogens and conjugated immunogens}

Fig. 1 shows the structures of the immunogens used for the in vivo immunisation procedures, i.e. 5hydroxybenzimidazole carbamate showing the site of conjugation of cBSA, 5-benzimidazolecarboxylic acid and 5-benzimidazolecarboxylic acid/Pam ${ }_{3}$ Cys$\mathrm{T}_{\mathrm{H}}$. 
<smiles>CC(=O)Nc1nc2cc(O)ccc2[nH]1</smiles>

5-Hydroxybenzimidazole carbamate

showing site of conjugation by EDC chemistry to $\mathrm{CBSA}$ via the $\mathrm{COOH}$ bond<smiles>O=C(O)c1ccc2[nH]cnc2c1</smiles>

5-Benzimidazolecarboxylic acid free unconjugated drug

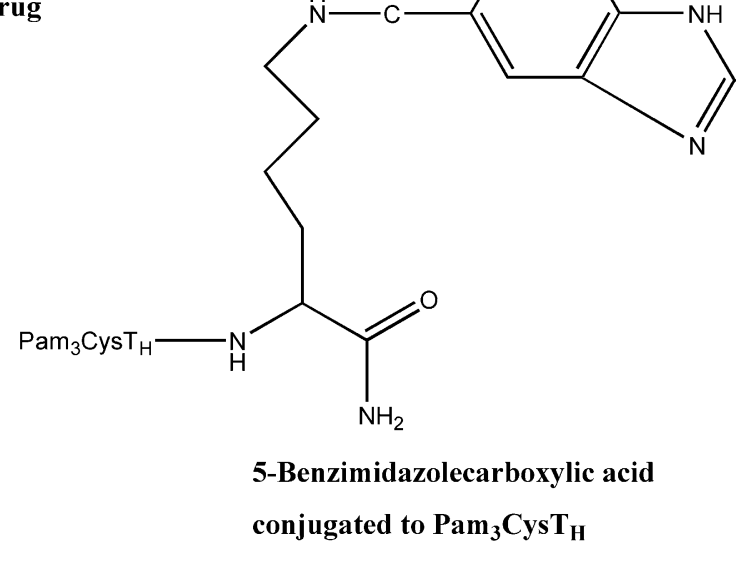

Fig. 1. Drugs and drug conjugates used as immunogens for in vivo immunisation procedures 1,2 and 3.

Fig. 2a shows the structures of the free drugs used for the in vitro immunisation regime, i.e. TBZ, 5hydroxythiabendazole and benzimidazole. Fig. 2b shows the control "capping" drug 10-hydroxydecanoic acid. Fig. 2c shows 2-benzimidazolepropionic acid, which, along with 5-benzimidazolecarboxylic acid, was used for screening hybridoma supernatants resulting from in vitro immunisation.

\subsection{Conjugation of haptens to carrier molecules}

Assessment of the EDC conjugation method was done by spectrophotometry, with a major peak seen at $300 \mathrm{~nm}$ (characteristic of 5-hydroxybenzimidazole carbamate) for conjugated cBSA/5-hydroxybenzimi- dazole carbamate which was absent in the spectrum scan for cBSA/EDC alone.

The calculated molecular mass of the 5-benzimidazolecarboxylic acid/Pam ${ }_{3} \mathrm{Cys}_{\mathrm{H}} \mathrm{T}_{\mathrm{H}}$ conjugate was 3064 .

3.3. Fusion/screening results for the in vivo immunisation regimes

\subsubsection{Immunogen 1: 5-hydroxybenzimidazole carbamate/cBSA}

Fusion of spleen cells immunised with cBSAconjugated 5-hydroxybenzimidazole carbamate and SP2 myeloma cells showed a fusion efficiency of $93 \%$ ( $\sim 360$ positive wells). Screening of resultant 
<smiles>c1ccc2[nH]c(-c3cscn3)nc2c1</smiles><smiles>Oc1ccc2[nH]c(-c3cscn3)nc2c1</smiles>

Thiabendazole (TBZ)<smiles>c1ccc2[nH]cnc2c1</smiles>

5-hydroxy-thiabendazole

Benzimidazole

(b)<smiles>O=C(O)CCCO</smiles>

(c)<smiles>O=C(O)CCC1=Nc2ccccc2C1</smiles>

2-Benzimidazolepropionic acid

Fig. 2. (a) Free drugs used as immunogens for in vitro immunisation regime. (b) The drug 10-hydroxydecanoic acid used for "capping" NH sites on CovaLink ELISA plates. (c) The additional drug used for screening supernatants from the in vitro immunisation regime.

hybridoma supernatants by ELISA method indicated approximately $10 \%$ positive hybridomas. Moderate drug-specific binding was observed for all the positive samples. This was indicated by moderate differences between $A_{405}$ readings obtained for supernatants screened against cBSA-conjugated 5-hydroxybenzimidazole carbamate and $A_{405}$ readings obtained for negative controls.

\subsubsection{Immunogen 2: admixture of drugs $/ \mathrm{Pam}_{3} \mathrm{Cys}-\mathrm{T}_{H}$}

Thirteen days following the fusion of immunised spleen cells with SP2 myeloma cells, $>350$ supernatants ( $91 \%$ fusion efficiency) were screened against 5-benzimidazolecarboxylic acid immobilised on CovaLink NH. Supernatants from all hybridomas showing positivity in wells with a drug present and a significantly lower reading in negative control wells were chosen for expansion and further testing. Eight hybridomas were expanded and three of these, upon expansion and repeated screening, continued to show significant differences in $A_{490}$ readings between positive and negative wells as shown in Table 1.
Supernatants from the three hybridomas above plus the positive and negative control polyclonal sera were assessed for their binding capacity to 5-benzimidazolecarboxylic acid by BIACORE analysis. Using this method of screening, very low binding responses were

Table 1

$A_{492}$ values for three selected supernatants when screened on CovaLink ELISA plates against the hapten 5-benzimidazolecarboxylic acid and the negative control drug, 10-hydroxydecanoic acid

\begin{tabular}{lll}
\hline Supernatant & $\begin{array}{l}A_{492} \\
\text { 5-benzimidazolecarboxylic } \\
\text { acid }\end{array}$ & $\begin{array}{l}A_{492} \\
10 \text {-hydroxydecanoic } \\
\text { acid }\end{array}$ \\
\hline $4 \mathrm{~F} 4$ & 1.320 & 0.178 \\
$8 \mathrm{~A} 7$ & 0.862 & 0.221 \\
$8 \mathrm{E} 2$ & $>2.00$ & 0.880 \\
$+\mathrm{ve}^{\mathrm{a}}$ & 0.737 & 0.055 \\
$-\mathrm{ve}^{\mathrm{b}}$ & 0.130 & 0.021 \\
\hline
\end{tabular}

${ }^{a}+$ ve control = polyclonal serum from mouse immunised with an admixture of drugs $/ \mathrm{Pam}_{3}$ Cys- $\mathrm{T}_{\mathrm{H}}$ (1/1000 dilution in CovaBuffer).

b - ve control $=$ polyclonal serum from nonimmunised mouse (1/1000 dilution in CovaBuffer). 
seen with all the samples including the positive control sample (average response unit (RU) was 50-60 RU). These responses were considered insignificant.

\subsubsection{Immunogen 3: 5-benzimidazolecarboxylic acid/} $\mathrm{Pam}_{3} \mathrm{Cys}-\mathrm{T}_{H}$ conjugate

A total of 300 supernatants (approximately 80\% fusion efficiency) were screened using both ELISA methods. Definitive results were obtained only when tested supernatants were screened by the MaxiSorp ELISA method, i.e. with 5-benzimidazolecarboxylic acid $/ \mathrm{Pam}_{3} \mathrm{Cys}_{\mathrm{H}} \mathrm{T}_{\mathrm{H}}$ or with $\mathrm{Pam}_{3} \mathrm{Cys}-\mathrm{T}_{\mathrm{H}}$ alone bound to wells. $18 \%$ of supernatants showed significantly higher $A_{405}$ readings in wells with bound 5-benzimidazolecarboxylic acid/ $\mathrm{Pam}_{3}$ Cys- $\mathrm{T}_{\mathrm{H}}$ compared to wells with $\mathrm{Pam}_{3} \mathrm{Cys}_{\mathrm{H}} \mathrm{T}_{\mathrm{H}}$ alone (Table 2). Supernatants from

Table 2

$A_{405}$ values for the selected supernatants when screened on MaxiSorb ELISA plates against the conjugate 5-benzimidazolecarboxylic acid/Pam ${ }_{3}$ Cys- $\mathrm{T}_{\mathrm{H}}$ and $\mathrm{Pam}_{3}$ Cys- $\mathrm{T}_{\mathrm{H}}$ alone

\begin{tabular}{|c|c|c|}
\hline Supernatant & $\begin{array}{l}A_{405} \\
\text { 5-benzimidazolecarboxylic } \\
\text { acid/Pam }_{3} \text { Cys-T }_{\mathrm{H}}\end{array}$ & $\begin{array}{l}A_{405} \\
\operatorname{Pam}_{3} \mathrm{Cys}_{\mathrm{H}} \mathrm{T}_{\mathrm{H}}\end{array}$ \\
\hline $1 \mathrm{C} 3$ & 0.524 & -0.031 \\
\hline $1 \mathrm{C} 7$ & 0.613 & -0.046 \\
\hline $1 \mathrm{~F} 2$ & 0.534 & 0.095 \\
\hline $2 \mathrm{~A} 4$ & 0.507 & -0.046 \\
\hline $2 \mathrm{~A} 5$ & 0.511 & -0.058 \\
\hline $2 \mathrm{~A} 7$ & 0.595 & -0.046 \\
\hline $2 \mathrm{~B} 6$ & 0.597 & -0.021 \\
\hline $2 \mathrm{C} 4$ & 0.510 & -0.002 \\
\hline $2 \mathrm{~F} 2$ & 0.569 & -0.015 \\
\hline 2D5 & 0.753 & -0.028 \\
\hline $3 \mathrm{~A} 8$ & 0.502 & -0.021 \\
\hline 3D5 & 0.527 & -0.021 \\
\hline $3 \mathrm{~F} 8$ & 0.564 & -0.020 \\
\hline $5 \mathrm{E} 1$ & 0.558 & -0.013 \\
\hline 7A5 & 0.474 & -0.011 \\
\hline 7B6 & 0.429 & -0.029 \\
\hline 7E8 & 0.622 & 0.002 \\
\hline 8D1 & 0.615 & 0.022 \\
\hline $8 \mathrm{E} 3$ & 0.565 & 0.008 \\
\hline $8 \mathrm{E} 4$ & 0.635 & -0.027 \\
\hline$+\mathrm{ve}^{\mathrm{a}}$ & 0.726 & -0.005 \\
\hline$-v e^{b}$ & 0.067 & -0.037 \\
\hline
\end{tabular}

20 wells showing $A_{405}$ readings close to or above 0.500 were selected for further analysis, including drug inhibition studies, using the BIACORE 3000 System.

By BIACORE analysis, nine of the supernatants showed low (an average of 1235 RU value was obtained) but consistent RU values which were decreased 3- to 4-fold (reduced to an average of $358 \mathrm{RU}$ ) by the addition to the test supernatants of free 5-benzimidazolecarboxylic acid. These additional studies indicated specific recognition of the drug by the antibody present in the supernatants and specificity for the drug was the paramount concern of this study. Positive and negative controls were the same as employed for the previous BIACORE screening.

\subsection{Fusion/screening results for in vitro immunisation protocols}

3.4.1. Immunogen 4: combination of free drugs, i.e. TBZ, 5-hydroxythiabendazole and benzimidazole

Following in vitro immunisation of BALB/c mice spleen cells and fusion of immunised spleen cells with SP2 myeloma cells, approximately 300 supernatants ( $\sim 80 \%$ efficiency) were screened for reactivity against 5-benzimidazolecarboxylic acid and 2-benzimidazolepropionic acid by ELISA using CovaLink NH ELISA plates.

Approximately 40 supernatants showed differential positivity against the screening compounds with the majority of the clones reacting more positive with 5benzimidazolecarboxylic acid than with 2-benzimidazolepropionic acid when compared with the negative control wells. The difference in $A_{492}$ between positive and negative control wells (approximately 1.6- to 2.7fold) was subsequently considered too low to warrant expanding the hybridomas or having the supernatants assessed by BIACORE analysis.

\section{Discussion}

The inherent problems associated with producing MAbs to small haptens are well documented and discussed (Clementi et al., 1991; Chappey et al., 1994; Danilova, 1994; Mittenbuhler et al., 1997; Tuomola et al., 2000). In an attempt to successfully 
produce hapten-specific MAbs, we have examined both in vivo and in vitro immunisation protocols using different methods of hapten conjugation to carrier molecules and various detection/screening methods.

The specific aim of the project was to produce MAbs that had the potential to recognise the proposed structure of protein-bound tissue residues of TBZ, i.e. possessing the phenyl ring of benzimidazole. To optimise the chances of producing such MAbs, we selected the most suitable drug molecules available that could be bound via bridging groups to carrier molecules while still allowing the phenyl ring of benzimidazole to be exposed as the major antigenic site. The information obtained from this work may be generally useful for the production of MAbs to a range of small molecules.

Of the immunisation methods employed, we found that the in vivo methodology, which was most successful in producing MAbs specific to the chosen target antigen (i.e., a drug molecule conjugated to a lipopeptide/T-cell epitope which acts as both a carrier molecule and an adjuvant), has been previously shown to have great potential for the production of MAbs to other non-immunogenic small haptens such as peptide drugs, antibiotics and toxins (Mittenbuhler et al.,1997; Hoffmann et al., 1997; Baier et al., 2000).

The most likely reasons why 5-benzimidazolecarboxylic acid conjugated to the lipopeptide $\mathrm{Pam}_{3}$ Cys$\mathrm{T}_{\mathrm{H}}$ was the most successful immunogen identified in this study were: (i) the lipopeptide Pam $_{3}$ Cys- $\mathrm{T}_{\mathrm{H}}$ carrier molecule had a much longer side chain than that used for the conjugation of the cBSA to 5hydroxybenzimidazole carbamate which in turn probably gave rise to more hapten-specific antibodies, (ii) the selection of the carrier molecule and method/s of conjugation of the immunogens is paramount for the successful production of specific MAbs. The Pam $_{3}$ Cys- $T_{H}$ has been previously shown to be more effective in the production of specific anti-hapten MAbs than the conventional carrier molecules such as BSA or KLH. Small molecules, even when carrying a B- and T-cell epitope, very often show low immunogenicity that is mainly due to the rapid breakdown of the molecule in vivo or clearance via the renal pathway. The use of this novel carrier molecule/adjuvant system apparently overcomes this limitation (Hoffmann et al., 1997; Baier et al., 2000).

Since we had already successfully produced MAbs to relatively short peptide sequences (12aa and a combination of 11 and 15 aa residues) conjugated to the conventional carriers BSA and KLH in vivo (Moran et al., 1997; Connolly et al., 2001), one of the other in vivo immunisation methods investigated in this study involved conjugation of 5-hydroxybenzimidazole carbamate to the modified and cationised BSA carrier protein (cBSA) via a carboxyl group attached to the imidazole group. In theory, cBSA, because of its positive charge, is believed to have a greater affinity for the negatively charged cell surface membrane of the antigen-presenting cells (APC), resulting in more efficient processing and presentation of the antigen and an enhanced immune response (Apple et al., 1988). The resultant low-level drugspecific MAbs produced may have been associated with the relatively short length of the linker arm on which, for conjugation purposes, the carboxyl group was located. Tuomola et al. (2000) have previously shown that haptens with short bridging groups failed to elicit hapten-specific antibodies but the same hapten with longer bridging groups produced antihapten responses. A possible explanation cited for this is that when a hapten is linked to the carrier protein by a short linker group, it is masked or lost within the protein tertiary structure, but if the linker is sufficiently long, it can project the hapten above the carrier protein surface (Paxton et al., 1976). It has been shown by Chappey et al. (1994) in a study of the association constants $\left(K_{\mathrm{a}}\right)$ for haptens with molecular weights ranging from 111 to $1202 \mathrm{Da}$ that haptens in the range of 334-374 Da result in high $K_{\mathrm{a}}$ values, but if the molecular weight of the hapten is under $300 \mathrm{Da}$, the probability of raising MAbs with high affinity is greatly diminished and that the influence of the linkage arm to the carrier protein is a very important consideration.

From the results of the in vivo immunisation procedure using immunogen 2, it would appear, however, that the presence of the lipopeptide $\mathrm{Pam}_{3} \mathrm{Cys}_{\mathrm{H}}$, acting basically as an adjuvant, was not sufficient to produce specific anti-hapten antibodies in the case of the very small haptens used as immunogens in this study. Instead, conjugation of the hapten to the carrier lipopeptide $\mathrm{Pam}_{3} \mathrm{Cys}_{\mathrm{H}} \mathrm{T}_{\mathrm{H}}$ was 
necessary to achieve the desired outcome of specific anti-drug antibodies. Using the lipopeptide $\mathrm{Pam}_{3}$ Cys$\mathrm{T}_{\mathrm{H}}$ as an adjuvant only appeared to produce specific target drug antibodies as indicated by the results obtained using the ELISA screening method. However, these results were not confirmed when a small number of selected supernatants were tested using the BIACORE 3000 system.

Employing a combination of three unconjugated drugs and an in vitro immunisation procedure, antibodies with weak binding to the target screening antigens (i.e., 5-benzimidazolecarboxylic acid and 2benzimidazolepropionic acid) were obtained when binding to the negative control drug 10-hydroxydecanoic acid was taken into account. Since we have successfully produced on a previous occasion specific MAbs using a 12-amino acid peptide conjugated to BSA in an in vitro immunisation protocol (Larkin et al., 1999), the effect of using an admixture of lipopeptide and drug or $\mathrm{Pam}_{3} \mathrm{Cys}_{\mathrm{S}} \mathrm{T}_{\mathrm{H}}$-conjugated drug warrants further investigation for the in vitro production of drug-specific MAbs. Hoffman et al. (1990) reported that lipopeptides can enhance the primary immune response when administered simultaneously with or conjugated to the antigen. Also, the obvious advantages of using the in vitro system (i.e., very short lead time from initial immunisation to final $\mathrm{MAb}$ production and elimination of the possibility of secretion of the hapten by the renal pathway) have to be weighed against the possibility of subsequently obtaining predominantly IgM subclass antibodies.

From these studies, it is clear also that the choice of screening method used to identify anti-target drug MAbs in supernatants from hybridomas is important and needs to be carefully considered. The two ELISAbased screening methods used during this study (i.e., CovaLink NH ELISA or hapten/carrier molecule conjugate bound to MaxiSorp ELISA plates) gave quite different results when supernatants resulting from the various immunisation regimes were screened. In the case of the hybridoma supernatants resulting from the fusion of spleen cells immunised with 5-benzimidazolecarboxylic acid conjugated to the lipopeptide $\mathrm{Pam}_{3} \mathrm{Cys}-\mathrm{T}_{\mathrm{H}}$, conclusive results were obtained when supernatants were screened against the conjugated hapten/carrier molecule and the carrier molecule alone on MaxiSorp ELISA plates. However, no conclusive results were obtained when the same supernatants were screened against 5-benzimidazolecarboxylic acid covalently bound to CovaLink $\mathrm{NH}$ plates and weak but apparently specific results (confirmed further by drug binding inhibition studies) were obtained when the screening of selected clones was done by BIACORE analysis.

In conclusion, this study has shown that the use of conventional adjuvant systems and carrier molecules conjugated to very small haptens may not be optimal for the generation of specific anti-hapten MAbs. The use and development of alternative carrier molecules/adjuvant systems that maintain the integrity of the immunogen in vivo appear to be very useful for the successful production of anti-hapten MAbs.

\section{Acknowledgements}

The authors wish to acknowledge the financial support of this work from the Department of Agriculture, Food and Rural Development under the Non-Commissioned Food Research Programme, Project Reference Number 98/R and D/T/199. The expert advice and assistance with animal handling of the staff at the Biomedical Research Facility, Beaumont Hospital, Dublin, Ireland, are also greatly acknowledged.

\section{References}

Apple, R.J., Domen, P.L., Muckerheide, A., Michael, J.G., 1988. Cationization of protein antigens: IV. Increased antigen uptake by antigen-presenting cells. J. Immunol. 140, 3290.

Baier, W., Loleit, M., Fischer, B., 2000. Generation of antibodies directed against the low-immunogenic peptide-toxins microcystin-LR/RR and nodularin. Int. J. Immunopharmacol. 22 (5), 339.

Brandon, D.L., Binder, R.G., Bates, A.H., Montague Jr., W.C., 1992. Monoclonal antibody-based ELISA for Thiabendazole in liver. J. Agric. Food Chem. 40, 1722.

Chappey, O.N., Sandouk, P., Scherrmann, J.M.G., 1992. Monoclonal antibodies in hapten immunoassays. Pharm. Res. 9 (11), 1375.

Chappey, O., Debray, M., Niel, E., Scherrmann, J.M., 1994. Association constants of monoclonal antibodies for hapten: heterogeneity of frequency distribution and possible relationship with hapten molecular weight. J. Immunol. Methods 172, 219.

Clementi, M.E., Marini, S., Condo, S.G., Giardina, B., 1991. Antibodies against small molecules. Ann. Ist. Super. Sanita 27 (1), 139. 
Connolly, L., Moran, E., Larkin, A., Scheffer, G., Scheper, R., Sarkadi, B., Kool, M., Clynes, C., 2001. A new monoclonal antibody, P2A8(6), that specifically recognises a novel epitope on the multidrug resistance-associated protein 1 (MRP1), but not on MRP2 nor MRP3. Hybridoma Hybridomics 20 (5), 333.

Coulet, M., Eechoutte, C., Larrieu, G., et al., 1998a. Comparative metabolism of Thiabendazole in cultured hepatocytes from rats, rabbits, calves, pigs and sheep, including the formation of bound residues. J. Agric. Food Chem. 46, 742.

Coulet, M., Dacasto, M., Eeckhoutte, C., et al., 1998b. Identification of human and rabbit cytochromes $P 4501 \mathrm{~A} 2$ as major isoforms involved in Thiabendazole 5-hydroxylation. Fundam. Clin. Pharmacol. 12, 225.

Danilova, N.P., 1994. ELISA screening of monoclonal antibodies to haptens: influence of the chemical structure of hapten-protein conjugates. J. Immunol. Methods 173, 111.

Fasciglione, G.F., Marini, S., Bannister, J., Giardina, B., 1996. Hapten-carrier interactions and their role in the production of monoclonal antibodies against hydrophobic haptens. Hybridoma 15 (1), 1.

Hoffman, P., Jimenez-Diaz, M., Loleit, M., Troger, W., Wiesmuller, K.H., Metzger, J., et al., 1990. Preparation of human and murine monoclonal antibodies: antigens combined with or conjugated to lipopeptides constitute potent immunogens for in vitro and in vivo immunisations. Hum. Antib., Hybridomas 1 (3), 137.

Hoffmann, P., Loleit, M., Mittenbuhler, K., et al., 1997. Induction of an epitope-specific humoral immune response by lipopeptidehapten conjugates: enhancement of the anti-melittin response by a synthetic T helper (Th)-cell epitope. FEMS Immunol. Med. Microbiol. 17 (4), 225.

Holley, M., 1998. In vitro production of monoclonal antibodies. Cell Biology: A Laboratory Handbook, 2nd ed. Academic Press, p. 404.

Kohler, G., Milstein, C., 1975. Continuous cultures of fused cells secreting antibody of predefined specificity. Nature 256, 495.
Larkin, A.M., Moran, E., Alexander, D., Doherty, G., Clynes, M., 1999. A new monoclonal antibody that specifically recognises the $m d r-3$ encoded gene product. Int. J. Cancer 80, 265.

Mittenbuhler, K., Loleit, M., Baier, W., et al., 1997. Drug specific antibodies: T-cell epitope-lipopeptide conjugates are potent adjuvants for small adjuvants in vivo and in vitro. Int. J. Immunopharmacol. 19, 277.

Moran, E., Larkin, A.M., Doherty, G., Kelehan, P., Kennedy, S., Clynes, M., 1997. A new $m d r$-1-encoded p-170 specific monoclonal antibody: (6/1C) on paraffin wax embedded tissue without pretreatment of sections. J. Clin. Pathol. 50, 465.

Moran, E., Larkin, A., Masterson, A., Clynes, M., 1998. Generation of monoclonal antibodies and characterisation of novel antibodies by Western blotting and immunocytochemistry. In: Clynes, M. (Ed.), Animal Cell Culture Techniques, Springer Lab Manual. Springer, Berlin, p. 82 .

Nunc Technical Literature, 2000. Covalent coupling of peptides to a CovaLink $^{\mathrm{TM}} \mathrm{NH}_{2}$ primary amine surface. TechNote 4 (27), 1-3.

Paxton, J.W., Rowell, F.J., Radcliffe, J.G., 1976. Production and characterisation of antisera to diphenylhydantoin suitable for radioimmunoassay. J. Immunol. Methods 10, 317.

Quinn, J.G., O'Kennedy, R., Smyth, M., Moulds, J., Frame, T., 1997. Detection of blood group antigens utilising immobilised antibodies and surface plasmon resonance. J. Immunol. Methods $206,87$.

Staros, V.V., Wright, R.W., Swingle, D.M., 1986. Enhancement by $\mathrm{N}$-hydroxysulfosuccinimide of water soluble carbodimide-mediated coupling reactions. Anal. Biochem. 156, 220.

Tuomola, M., Harpio, R., Mikola, H., Knuuttila, P., Lindstrom, M., Mukkala, V.M., Matikainen, M.T., Lovgren, T., 2000. Production and characterisation of monoclonal antibodies against a very small hapten, 3-methylindole. J. Immunol. Methods 240, 111. 